DOCTRINA

\title{
La discriminación estructural de género y su recepción sistémica en el sistema de derechos humanos
}

\author{
Structural gender discrimination and its systemic understanding \\ in the human rights system
}

\author{
Claudia Iriarte Rivas \\ Universidad de Chile
}

\begin{abstract}
RESUMEN Este trabajo recoge el planteamiento iusfeminista de la necesidad de introducir en el derecho internacional de los derechos humanos de un modo sistemático el enfoque de discriminación estructural de género como un mecanismo que permita abordar la realidad material del sujeto mujeres, dar cuenta del conflicto social de género, a fin de avanzar en la superación de la discriminación sistémica que afecta a las mujeres en materia de derechos. Para ello revisaremos, en primer lugar, la manera en que en el naciente derecho de los derechos humanos se recibió la noción abstracta de sujeto configurada por el constitucionalismo. Luego se verá cómo esta concepción, aún presente como sustrato del derecho internacional de los derechos humanos, ha impedido que los avances sucesivos del derecho internacional de los derechos humanos respecto de las mujeres constituyan modificaciones sustantivas. También analizaremos de qué modo la recepción de la noción de discriminación estructural permitiría avanzar de un modo importante en la deconstrucción de la noción de sujeto inicial y en la construcción de una concepción que recoja la subjetividad de los diversos sujetos y su materialidad.
\end{abstract}

PALABRAS CLAVE Derechos humanos, iusfeminismo, sujeto jurídico.

ABSTRACT This article is based on the feminist legal studies concept, which states the need to introduce in the international human rights systematically the idea of structural gender discrimination as a mechanism to approach the material reality of women as subjects and emphasizes the social gender conflict to make a step in overcoming the systemic legal discrimination women are exposed to. In the first place we will analyze the way in which was taken up the abstract notion of the subject configured by the constitutionalism. Then we will analyze how this concept, still present as a substrate of the International Human Rights, has impeded that the ongoing progress in the Interna- 


\begin{abstract}
tional Human Rights referred to women gives way to substantial changes. We also analyze how the understanding of the concept of structural discrimination might facilitate the deconstruction of the concept of the initial subject and the construction of a concept which takes into account the subjectivity of diverse subjects and their materiality.
\end{abstract}

KEYWORDS Human rights, feminist legal studies, legal subject.

\title{
Introducción
}

El iusfeminismo sostiene que la discriminación que afecta a las mujeres no puede abordarse con las herramientas tradicionales fundadas en la concepción de aplicación injusta de la norma, sino desde la conceptualización de la discriminación sistémica. El sistema de los derechos humanos, si bien ha tenido una inflexión importante en la concepción de discriminación de las mujeres desde la dictación de la Convención sobre la Eliminación de Todas las Formas de Discriminación contra la Mujer (CETFDCM, también conocida por sus siglas en inglés: CEDAW), aún no logra configurar de un modo estructural una noción de discriminación que exprese la discriminación sistémica que afecta al sujeto mujeres.

De modo central, las limitaciones del abordaje de la discriminación de género están determinadas por el constitucionalismo, el cual expresó la construcción jurídica de la Modernidad y específicamente la configuración y construcción efectuada a partir de la noción de sujeto abstracto.

Para analizar este problema y la necesaria consideración de la discriminación estructural a fin de avanzar de modo más efectivo en la superación de la discriminación en materia de derechos, revisaremos primeramente la manera en que en el naciente derecho de los derechos humanos se recibió sin un planteamiento crítico la noción abstracta del constitucionalismo, para luego analizar la implicancia que tuvo la recepción del sujeto abstracto en la configuración de los derechos. También se verá cómo esta concepción, aún presente como sustrato del derecho de los derechos humanos, impide que los cambios y los avances sucesivos del derecho internacional de los derechos humanos respecto de las mujeres se manifiesten a cabalidad. Finalmente, se analizará cómo la recepción de la noción de discriminación estructural permitiría avanzar de un modo importante en la deconstrucción de la noción de sujeto inicial y en la construcción de una concepción que recoja la subjetividad de los diversos sujetos y su materialidad.

\section{Noción de persona}

El constitucionalismo configurado en Occidente constituye uno de los pilares fundamentales en la construcción conceptual de la Modernidad y de la democracia, y, 
por lo mismo, una parte esencial de la institucionalidad jurídico-política actual está determinada por la forma en que se diseñó y configuró el constitucionalismo. ${ }^{1}$

Esta nueva institucionalidad expresó (y expresa) su concordancia con los principios recibidos del racionalismo en dos planos. En primer lugar, configura un modelo de norma jurídica racional -la Constitución - que estructura y ordena el poder político de un modo unitario, sistemático y no contradictorio. Esta norma comprende, por una parte, el reconocimiento de los derechos de los ciudadanos, los que están, a su vez, concebidos como fines y límites del Estado, y por otra, la regulación de las funciones fundamentales del Estado y de los órganos que lo integran.

En segundo lugar, es concordante con los principios del racionalismo, en cuanto a la concepción del poder político que es recibida en el orden constitucional. De esta manera, se configura una estructura basada en los postulados y principios iusracionalistas que determinarán la ordenación del poder político, dentro de la cual se establecerán las instituciones propias del Estado.

La recepción del ideario racionalista requiere la configuración de un modelo jurídico construido sobre nociones abstractas que no tienen materialidad en una realidad concreta, dando respuesta a las necesidades teóricas derivadas del modelo explicativo de configuración de la sociedad política.

Por ello, de un modo central se requería configurar una noción general y abstracta de persona (sujeto jurídico) que permitiera responder a la nueva conceptualización del sujeto político, el cual se constituyó como portador de derechos, y para esto fue necesario superar el concepto de individuo físico por el de la persona moral. El origen del constitucionalismo es también el origen del sujeto individual. El profesor Ermanno Vitale plantea la idea del vínculo entre derechos y noción de individuo, señalando: «para pensar el objeto jurídico 'derecho fundamental' resulta necesario [...] tener una idea de individuo o de persona [...] Pero quizás haya más, del individuo o de la persona resulta necesario definir sus predicados esenciales [...] La naturaleza del individuo y de sus relaciones no es indiferente para la definición no sólo de cuáles son los derechos fundamentales, sino del propio concepto de derecho fundamental» (Vitale, 2009: 69).

La concepción abstracta jurídica de persona, que estaba en construcción, debía ser concordante con los postulados que el racionalismo tenía sobre la persona humana. Así, la persona debía ser racional, ${ }^{2}$ libre y soberana de sí misma.

1. Por constitucionalismo se entiende el movimiento jurídico que positiviza los postulados del jusracionalismo en textos normativos fundamentales. Estos textos regulan las condiciones del pacto que constituye la sociedad política; reconocen y garantizan los derechos fundamentales y norman los principios que organizan los poderes que se constituyen por el pueblo soberano, estructurando de esta forma al Estado. Sobre el concepto de constitucionalismo, véase Dippel (2009), Ferrajoli (2011a) y Hespanha (2002).

2. «La definición de hombre realizada por el cartesianismo era la del ser racional: ser que buscaba la verdad a través de la razón» (Hespanha, 2002: 154). 
La subjetivización del individuo abstracto del constitucionalismo se realizó sobre estas premisas del racionalismo, lo que significó que la noción de sujeto de la Modernidad comprendió al varón, libre, adulto, letrado y con patrimonio, subjetivando y determinando al sujeto político y al sujeto de derechos. Quienes no cumplían con la condición de ser racionales, libres y soberanos de su persona - es decir, las mujeres, los iletrados, los esclavos, los sirvientes domésticos, los obreros, los campesinos, los indígenas, los pródigos, los insolventes y los discapacitados- no fueron considerados en la subjetivización del sujeto abstracto racional.

La noción de persona fue recibida normativamente por el constitucionalismo, por lo que en las constituciones elaboradas a fines del siglo XVIII y durante el siglo XIX, la condición de ciudadano estaba reservada sólo a quienes quedaban comprendidos en la noción de persona. Los y las demás no eran ciudadanos, no eran sujetos de derecho y el reconocimiento de los derechos respecto de ellos y ellas era inexistente.

\section{Ampliación de la ciudadanía: ¿Cambios en la subjetivización del sujeto?}

El proceso de ampliación de ciudadanía de las mujeres (y su consiguiente extensión de derechos) fue un proceso lento e irregular. Se fue ampliando su capacidad jurídica laboral, primero, y luego civil, y en materia de ciudadanía política, esta se extiende mayoritariamente en Occidente luego de la Segunda Guerra Mundial. Los procesos de cambio en materia jurídica no se limitaron al ámbito de la ciudadanía política y capacidad. En este período, se desarrolló también un proceso de reformas al ordenamiento jurídico referentes a la regulación del matrimonio y la familia; se fueron modificando la potestad marital, la patria potestad, los regímenes patrimoniales del matrimonio, la regulación del divorcio, la regulación de los delitos sexuales, el aborto, el adulterio. Estos cambios se orientaron fundamentalmente a mejorar la condición jurídica de la mujer. Sin embargo, al igual que las reformas en materia de ciudadanía política, estas se diseñaron e implementaron sin modificar la estructura que consagraba un orden respecto del espacio público y privado, un rol social de la mujer, un orden de las familias, una jerarquía al interior de la familia y la asignación del lugar de hombres y mujeres en la esfera pública, privada y familiar. En una frase, sin modificar la estructura del orden sexual.

Ahora bien, cabe preguntarse qué significó el proceso de ampliación de la ciudadanía, respecto del sujeto abstracto configurado por la Modernidad. La noción jurídica de persona en lo formal se amplía, es verdad, la noción de sujeto ciudadano comprende ahora formalmente a las mujeres. Sin embargo, el modelo, que subyace al sistema de los derechos fundamentales, no se ha modificado y continúa configurado sobre la base de una noción de sujeto que no percibe a la mujer en su subjetividad. Como dice Fraisse (2003), las mujeres dejaron de estar excluidas para pasar a estar discriminadas. Se produce así una ambivalencia, el reconocimiento de ciudada- 
nía (política, social y económica), que abarcando la capacidad de obrar le otorgó existencia en la sociedad política y en el mercado, dejando atrás la exclusión, no la hace un igual; por ello, el derecho de igualdad respecto de la mujer continúa siendo declarativo.

La institucionalidad jurídica dio cuenta del sujeto mujeres considerándolo como la alteridad del sujeto ya conceptualizado. ${ }^{3}$ La noción de sujeto no se deconstruye, solo se le agrega algo. Lo femenino se considera como un otro específico, cuyas particularidades complementan el concepto ya existente. Como señala Habermas:

Las diferencias entre los sexos no se entienden como relaciones necesitadas de interpretación y susceptibles de interpretación entre dos magnitudes de referencia igualmente problemáticas, sino que se entienden como desviaciones respecto del prototipo de «situación normal», es decir, de [una] situación cortada a la medida de los varones (Habermas, 2008: 509).

Uno de los casos más significativos es la legislación en materia laboral: más que como trabajadoras, fueron consideradas mujeres a las que debía protegerse por su minusvalía y su sujeción a la familia (Pateman, 2000). De esta manera, se limitó su posibilidad de realizar determinados trabajos y faenas (prohibición del trabajo minero, nocturno y donde se expusieran a actividades licenciosas), ${ }^{4}$ se les protegió en su condición de madres y se las amparó como cónyuges de los trabajadores. En otras palabras, a la mujer se le reconoce como trabajadora, pero sin que ello suponga cambiar su vínculo y pertenencia al ámbito de la familia, manteniendo la estructura de la división sexual del trabajo. ${ }^{5}$

\section{Realidad material del sujeto mujeres}

La doctrina iusfeminista cuestiona la configuración del sujeto jurídico/sujeto de derechos concebido como universal, en tanto fue configurada como una noción genérica que expresa al sujeto como una categoría neutra, que supera la diversidad.

Esta concepción del sujeto permite, a su vez, que el ordenamiento jurídico en su conjunto exprese esta abstracción ignorando la materialidad de los sujetos (De Cabo Martín, 2006: 43). Lo anterior implica abstraerse de la conflictividad social producto de las relaciones de poder que existen en el orden social. El modelo del constituciona-

3. La extensión de ciudadanía y capacidad de obrar respecto de los varones excluidos inicialmente de la ciudadanía implicó un cambio sustancial y no solo formal, ya que su subjetividad masculina ya estaba expresada en la concepción de sujeto jurídico.

4. Hubo legislación laboral que limitaba el trabajo de las mujeres donde tuvieran acceso a textos o dibujos licenciosos (cf. Arnaud-Duc, 1993).

5. En el Estado social, se reguló el salario familiar, que se definía como aquel se le pagaba al obrero, y que debía cubrir las necesidades de su núcleo familiar incluyéndose a su cónyuge. 
lismo liberal siguió el proceso de abstracción del racionalismo en lo referente al sujeto, construyendo la subjetividad individual sin considerar al sujeto real y su posición (o más bien sus posiciones) en la estructura social, disolviendo así la realidad social del sujeto en el orden jurídico, lo que implica ocultar las desigualdades y jerarquías entre los sujetos (Barcellona, 1996: 107). En el derecho de la Modernidad, las diferencias se han diluido y los individuos han entrado en una relación libre y autónoma donde intercambian esta libertad, abstrayéndose de las condiciones materiales de los sujetos en el momento del intercambio; el diseño de un modelo de derecho sobre la base de abstracción del sujeto dista en tal medida del sujeto material: se produce una especie de «vaciamiento» del sujeto (Barcellona, 1996: 123-126).

Respecto del sujeto mujeres, la noción de un sujeto abstracto significa ignorar la condición sexuada de los sujetos, cuyas vivencias y realidades están determinadas por la construcción de género. Esto, por su parte, supone invisibilizar las diferencias y subordinación, ${ }^{6}$ consolidando y validando las estructuras del sistema sexo género y ocultando, además, el conflicto social fundado en la diferencia sexual.

Histórica y ancestralmente los modelos patriarcales se han definido a partir del control sexual, del control reproductivo de las mujeres y/o de la apropiación del trabajo de las mujeres. Es en la Modernidad que este control se produce específicamente para poder garantizar la reproducción de la fuerza de trabajo, esto es, la reproducción biológica, que se asegura con el control de la sexualidad y capacidad procreativa de las mujeres, y la reproducción social, que se logra con el control y apropiación del trabajo gratuito de las mujeres en la economía del cuidado. ${ }^{7}$

El sistema sexo género determina lugares y roles sociales y relaciones de jerarquía y dominación entre los sujetos hombres y sujetos mujeres, constituyendo un conflicto social fundado en el sexo de los sujetos. El sistema sexo género se consagra y fija a través de un orden social sexual que se institucionaliza en el derecho, cimentándose y expresándose en el orden jurídico de la Modernidad.

La emancipación de los sujetos hombre que supone la Modernidad tiene su correlato en la sujeción de las mujeres en la esfera privada/doméstica. La afirmación de la libertad del individuo optimo iure implicaba a su vez la afirmación de su potestad dentro de la esfera privada familiar (Ferrajoli, 2004: 229; 2011a: 229). La esfera privada fue excluida del debate racional sobre la justicia (Rubio, 2006: 33) y, a su vez, de

6. Como dice Ferrajoli, «la(s) diferencia(s) de género [...] son desvalorizadas y negadas; en nombre de una abstracta afirmación de la igualdad» (2011b: 7).

7. Concepto elaborado por las economistas feministas que comprende el trabajo del cuidado y la producción de cuidado que se requiere para cubrir las necesidades humanas de reproducción biológica y social.

8. Noción utilizada por Ferrajoli para referirse a la noción de sujeto abstracto que es el sujeto jurídico/ sujeto de derecho del constitucionalismo y que expresa a un sujeto varón, blanco, propietario y letrado (Ferrajoli, 2004). 
la esfera política, lo que significó, y significa hasta hoy, que las relaciones de la esfera privada/doméstica no tienen un vínculo con el espacio público. La dicotomía pública/privada permite al Estado desentenderse de lo que sucede en el ámbito privado/ doméstico, despolitizando de paso las situaciones de menoscabo (discriminación estructural) que afectan al sujeto mujeres (Mestre, 2011: 148).

En el proceso de incorporación a la ciudadanía de las mujeres, ni el orden social sexual ni la estructura material de la familia fueron sometidos a procesos de revisión ni cambio en virtud de los nuevos requerimientos que trajo consigo el reconocimiento de la condición de ciudadana de la mujer. Por ello, aunque la mujer sea ciudadana, su ciudadanía es deficitaria; ya que mantiene, por una parte, su posición subordinada dentro de la jerarquía social, y, por otra, su subjetividad y realidad social no están presentes en el modelo de los derechos, ni en la noción de sujeto. Lo propio de su realidad material y condición de persona no es concordante con las condiciones para el pleno ejercicio de la ciudadanía y de los derechos.

Los factores históricamente invisibilizados aumentan su invisibilidad luego del reconocimiento de derechos a las mujeres, impidiendo que la desigualdad y el conflicto social que la origina se expresen en el orden jurídico político (De Cabo Martín, 2001: 123). Así, la discriminación de la mujer no es considerada como una discriminación producto del orden social, sino como el resultado de actuaciones individuales de sujetos. Por lo mismo, el no ejercicio en plenitud de las mujeres en materia de ciudadanía es considerado como expresión de una voluntad de estas, cuyo fundamento se encuentra en el modo de ser e intereses propios de las mujeres. Dicho de otro modo, la fundamentación del déficit de ciudadanía se construye por la vía de la naturalización de su realidad material.

Los cambios producidos por el reconocimiento de ciudadanía y capacidad jurídica de las mujeres han tensionado, cuestionado e incluso interferido el modelo construido en los albores de la Modernidad, pero sus bases fundamentales no han sido modificadas: continúan configuradas de espaldas a la diversidad sexual. Como señala Bodelón: «Dar cuenta del conflicto [derivado del orden sexual] significa que la discriminación debe abordarse como parte de un orden social que subordina a las mujeres y que no da cuenta de la diferencia de estatus que hay al interior de las sociedades» (2009: 231).

\section{El derecho de los derechos humanos: Noción de ser humano y universalidad de los derechos}

En la segunda mitad del siglo XX, los planteamientos, propuestas y nuevos órdenes normativos elaborados desde el derecho de los derechos humanos tuvieron (y tienen) particular importancia respecto de las nociones de ser humano, derechos fundamentales, universalidad e igualdad. En este desarrollo, los instrumentos internacionales 
cobran especial importancia la Declaración Universal de Derechos Humanos de 1948 y los pactos posteriores: el de derechos civiles y políticos y el de derechos económicos, sociales y culturales, aprobados en 1966. La elaboración, aprobación y posterior aplicación de estos textos, así como el debate doctrinario que acompañó este proceso, ha implicado planteamientos filosóficos jurídicos respecto de la persona humana y de la noción de los derechos.

Los derechos humanos se fundan en la idea de la universalidad de los atributos humanos, es decir, en la existencia de una condición intrínseca de la persona humana en cuanto ser humano, planteándose que se entiende como tal a quien tenga aspecto humano (le pertenecen los atributos humanos), independientemente de su condición o situación histórica o cultural (Dimier, 1985: 112).

El debate sobre la noción de derechos humanos plantea que estos son derechos que pertenecen al ser humano en cuanto tal. Los derechos humanos tienen sus raíces en la naturaleza humana, que es la misma en todas las épocas y lugares, es inmutable y universal, solo que está inserta en diferentes situaciones históricas y culturales (Mathieu, 1985: 35-36).

A partir de esta premisa, el debate se articula en la definición de los rasgos de la esencia del ser humano que lo convierten en sujeto de derechos o, dicho de otra forma, cómo debe concebirse la naturaleza humana para que exista sujeto de derecho. La condición de sujeto de derecho de los derechos humanos quedó definida por la capacidad - propia de la condición humana- de perseguir objetivos conscientemente (Mathieu, 1985: 119-120).

La concepción de fundamento de los derechos humanos en la institucionalidad del sistema de los derechos humanos se elaboró dentro de la concepción de universalidad de los derechos humanos de una noción universal de persona humana, instaurada en la Declaración Universal de 1948. La idea de universal — tanto del sujeto (todos los seres humanos), como de los derechos (todos los seres humanos son titulares) - significa que esta condición supera cualquier variable que implique limitar el carácter omnicomprensivo, sin distinción alguna, de todos los seres humanos como titulares iguales de todos los derechos humanos (Medina, 2006: 127). Por lo mismo, los derechos humanos son en sí aplicables respecto de cualquier ser humano, debiendo por parte del derecho garantizarse su ejercicio, goce y protección en igualdad de condiciones respecto de todos.

La noción de ser humano universal en el derecho de los derechos humanos se configura como un genérico que se constituye sobre la base de los atributos comunes de todos los seres humanos. Esta configuración del sujeto utiliza el modelo propio del racionalismo. El anclaje teórico en el racionalismo y constitucionalismo del derecho internacional de los derechos humanos, tanto en su origen y desarrollo posterior, se debe a que existe una continuidad teórico-normativa desde la configuración de los derechos en las nacientes constituciones hasta la etapa actual. Los derechos humanos 
se han configurado en un proceso de desarrollo por etapas que han sido acumulativas. Existen cuatro procesos que marcan el desarrollo de los derechos: la positivización, la generalización, la internacionalización y la especificación. ${ }^{9}$ La positivización se produjo en el proceso de recepción de los derechos y garantías en las constituciones; luego, la generalización supuso extender el alcance y contenido de los derechos a los sujetos que no los poseían; a su vez, la internacionalización implica el desarrollo de estos en el derecho internacional público; y la especificación consiste en abordar y considerar las particularidades de los sujetos o colectivos de sujetos.

Si observamos las etapas mencionadas es posible establecer que existe un continuum respecto del desarrollo de los derechos desde su positivización hasta la etapa de especificación, en el cual, de modo equivalente a lo ocurrido en las legislaciones domésticas (ya que no son más que expresión de un mismo proceso), no se desarrolló un proceso crítico respecto de la construcción y determinación del sujeto universal tributario de esos derechos ni tampoco respecto de la configuración de los derechos, su extensión y garantías, teniendo en consideración al sujeto universal.

En lo concerniente particularmente a derechos humanos y mujeres, la revisión de los instrumentos iniciales (comienzo del proceso de especificación) muestra que en 1946 se crea la Comisión de la Condición Jurídica y Social de la Mujer como mecanismo para plantear recomendaciones acerca de problemas que se presentan en relación con los derechos políticos, económicos, civiles, sociales y educativos de las mujeres. La Comisión se plantea como objetivo «elevar la condición de la mujer, independientemente de su nacionalidad, raza, idioma o religión, a la igualdad con los hombres en todos los ámbitos de la actividad humana, y eliminar todo tipo de discriminación contra la mujer en las disposiciones de la legislación, en las máximas legales o normas, o en la interpretación de la ley consuetudinaria» (ONU, 1947: 12). En 1952, se dicta la Convención sobre los Derechos Políticos de la Mujer, que tuvo por objetivo asegurar el ejercicio de los derechos políticos reconocidos en la Declaración Universal de Derechos Humanos de forma igualitaria. Posteriormente, en 1967 se dicta la Declaración sobre la Eliminación de la Discriminación Contra la Mujer, que insta a los países miembros a tomar medidas para garantizar el principio de igualdad de hombres y mujeres a través de la abolición de las leyes, reglamentos y costumbres que constituyan discriminación en contra de la mujer.

Como es posible observar, en los objetivos y fundamentos de estos instrumentos se plantea el logro de la igualdad de las mujeres y los hombres, objetivo que se pretende alcanzar a través de la supresión en el derecho de las discriminaciones que este contenga. En este instrumental, no solo aparece como idea de igualdad que las mujeres logren superar la discriminación por la vía de la igualdad con los hombres -lo que refuerza la noción androcéntrica de sujeto optimo iure-, sino que además

9. Este modelo ha sido desarrollado por varios autores. Cf. Peces-Barba (1995). 
no existe, ni en su fundamentación ni en sus objetivos ni en las herramientas planteadas, una revisión respecto de la subjetivización del sujeto (universal) tributario de los derechos, ni tampoco respecto de la configuración de los derechos (universales) y sus garantías.

En esta etapa, como no se realiza un análisis crítico respecto materialidad del sujeto mujeres, la normativa que se configuró como expresión de la especificación de derechos incluso refuerza el modelo social sexual y los estereotipos del sujeto femenino. En materia del derecho al trabajo, por ejemplo, se encuentran las normas referidas a la maternidad dirigidas a la madre trabajadora, sin considerar el planteamiento de la co-parentalidad, ni el principio de la responsabilidad social de la reproducción.

\section{Nuevos instrumentos internacionales}

En los últimos decenios del siglo XX se desarrollaron importantes propuestas respecto del sistema de los derechos, producto de un proceso de crítica y revisión del alcance, contenido y determinación de los derechos humanos. Este proceso crítico abarcó también la noción de ser humano, en cuanto a determinar si esta logra expresar la diversidad que la condición humana implica, y cómo a partir de ella es necesario configurar los derechos humanos universales de la persona humana. Este proceso crítico ha sido impulsado de un modo importante desde los movimientos de los derechos humanos (donde el movimiento feminista y sus propuestas han tenido importancia) y se ha nutrido de la elaboración doctrinaria que estos movimientos han construido o han influenciado.

La dictación de la CEDAW respecto de los derechos humanos y mujeres marcará un punto de inflexión en la línea planteada, ya que el objetivo fundamental de este texto, más que lograr la igualdad de hombres y mujeres, es eliminar las diversas formas de discriminación que afectan a las mujeres, recogiendo con ello la idea de que las mujeres en general constituyen un grupo social respecto del cual existe una realidad de exclusión basada en su sexo, del que resulta un menoscabo o desconocimiento de la titularidad, goce o ejercicio de los derechos humanos, en las diversas esferas de la sociedad. Además, esta Convención reconoce, en la propia definición de lo que entiende por discriminación contra la mujer, que se causará discriminación no solo cuando se tenga por objeto causarla (discriminación directa), sino también cuando se tenga por resultado una situación de discriminación (discriminación indirecta). ${ }^{10}$ A su vez, contempla que para la eliminación de la discriminación contra la mujer

\footnotetext{
10. La discriminación directa e indirecta son conceptos del derecho antidiscriminatorio. La primera se refiere a los tratamientos que sin razonabilidad desfavorecen a un determinado grupo; la segunda se refiere a normas de carácter neutral, cuya aplicación tiene igualmente el efecto de desfavorecer a un grupo determinado.
} 
es posible establecer medidas de acción positiva (artículo 4.1) de carácter temporal encaminadas a acelerar la igualdad de facto entre el hombre y la mujer. Son acciones que establecen una suerte de "ofertas de ventajas» (Barrére, 2001: 158-159) respecto del colectivo mujeres para superar la discriminación por la vía de mejorar las condiciones de inserción de ellas en diversas esferas de la vida social.

Sin embargo, la CEDAW tiene déficits importantes. Se puede señalar que la noción de discriminación establecida se sustenta en la defensa de los derechos y libertades desde una concepción aún formalista; esta sería una de las razones que explicaría por qué no fue posible que esta Convención abordara de modo expreso la violencia de género, considerando el carácter de discriminación sistémico que tiene, lo que sí ocurrió tiempo después en la Cuarta Conferencia Mundial de las Mujeres celebrada en Beijing en 1995, en la que se reconoce que violencia contra la mujer es «todo acto de violencia basado en el género» (ONU, 1995: párrafo 113). Además, este instrumento no se plantea la discriminación contra la mujer como un fenómeno producido por causas estructurales originadas en una materialidad de inferioridad y subordinación, ni tampoco la comprende como manifestación de relaciones de poder entre hombres y mujeres.

Con posterioridad a la CEDAW, hay dos declaraciones de Naciones Unidas referidas a la mujer que tienen especial importancia respecto del avance en el reconocimiento de sus derechos: la Declaración y el Programa de Acción de Viena (junio de 1993), y la Declaración sobre la Eliminación de la Violencia contra la Mujer (diciembre de 1993). ${ }^{11}$ La Declaración y el Programa de Acción de Viena establecen que los derechos humanos de las mujeres y las niñas forman parte inalienable e indivisible de los derechos humanos universales, ${ }^{12}$ lo que es sustancial en el proceso de subjetivización de la mujer respecto de la conceptualización de ser humano de los derechos humanos. La Declaración sobre la eliminación de la violencia contra la mujer de Naciones Unidas, en los considerandos reconoce «la existencia de relaciones de poder históricamente desiguales entre el hombre y la mujer, que han conducido a la dominación de la mujer y a la discriminación en su contra por parte del hombre e impedido el adelanto pleno de la mujer», lo que sin embargo no se plasma en la propia conceptualización de violencia, donde la violencia contra la mujer es definida «como todo acto de violencia basado en la pertenencia al sexo femenino» (ONU, 1993).

En este contexto, la dictación en el sistema interamericano de la Convención de

11. En el sistema de los derechos humanos, los tratados y costumbres constituyen las principales fuentes; sin embargo, otros tipos de fuentes en el ámbito internacional son cruciales para su interpretación y aplicación. Es importante tener presente declaraciones como las mencionadas; las declaraciones de las conferencias temáticas, así como las recomendaciones de los Comités, que han permitido ampliar la recepción de concepciones más innovadoras en materia de discriminación que permite superar el modelo formalista e individualista.

12. Específicamente su artículo 18 establece: «Los derechos humanos de la mujer y de la niña son parte inalienables, integrantes e indivisibles de los derechos humanos universales». 
Belem do Pará es un hito central en el derecho internacional de los derechos humanos respecto de los planteamientos iusfeministas, ya que es la única convención que ha recogido en su texto la perspectiva de la discriminación estructural en materia de violencia de género. Ahí se plantea que la violencia contra la mujer es una violación a sus derechos humanos y una «manifestación de las relaciones de poder históricamente desiguales entre mujeres y hombres»; para señalar, luego, en el artículo 6, el reconocimiento del derecho a una vida libre de violencia, el cual incluye el derecho de la mujer a ser valorada y educada libre de patrones estereotipados de comportamiento y prácticas sociales y culturales basadas en conceptos de inferioridad o subordinación. Este instrumento ha sido fundamental en las sentencias de la Corte Interamericana, ya que ha permitido avanzar en la consideración de la subjetividad de las mujeres para analizar y determinar la ocurrencia de discriminación de género. ${ }^{13}$

En esta etapa del proceso de especificación ha habido avances importantes en la recepción de la subjetividad de las mujeres. Sin embargo, este proceso ha sido dispar y parcial, y no se ha efectuado sistemáticamente la recepción y rearticulación de la institucionalidad de los derechos humanos referidos al sujeto mujeres. Como consecuencia de lo señalado, en materia de subjetivización de los derechos hay importantes déficits; es el caso del trabajo del cuidado realizado por las mujeres en la esfera doméstica, el cual no es considerado ni abordado por el sistema de derechos. Es posible también señalar el caso de la prohibición de la violencia de género (en espacios públicos y privados), que no ha sido abordada integralmente como derecho por el sistema de derechos humanos. En materia de ciudadanía política, la paridad de la representación pública de los sujetos tampoco se ha planteado en los instrumentos vinculantes. Un área que no es posible dejar de mencionar son los derechos sexuales y reproductivos; si bien en materia internacional de los derechos humanos, ha habido a este respecto un avance importante, luego de la sistematización de estos derechos en el Plan de la Conferencia Internacional sobre la Población y el Desarrollo de 1994, ${ }^{14}$ estos no han logrado constituirse como un corpus orgánico, coherente

13. En el caso del «campo algodonero», la Corte Interamericana de Derechos Humanos consideró que en Ciudad Juárez existía un patrón sistemático de violencia contra las mujeres, lo que significa que los homicidios ocurridos bajo ese patrón pueden ser catalogados de crímenes seriales y de violencia sexual influenciados por una cultura de discriminación contra la mujer, lo que es fundamental para catalogar los hechos de violencia de género, ya que, como recuerda la propia Corte, no toda violación de un derecho humano cometido en perjuicio de una mujer conlleva necesariamente una situación de violencia de género. Además, la determinación de violencia contra la mujer por razones de género permite a la Corte establecer que estos homicidios cometidos son femicidios, esto es, homicidios contra mujeres que son expresión de una concepción de inferioridad y subordinación de las mujeres. Véase CIDH, Caso González y otras contra México ("Campo Algodonero»), sentencia del 16 de noviembre de 2009, serie c, núm. 205, párr. 143.

14. Disponible en http://bit.ly/2DvYW30. 
y sistemático que permita superar el carácter eminentemente precario ${ }^{15}$ que tienen hasta ahora.

\section{La noción de discriminación estructural y los derechos humanos}

Hemos dicho que la noción de sujeto jurídico configurada en la Modernidad y recibida por el constitucionalismo se funda en una construcción de sujeto abstracto, universal y que, posteriormente, los derechos humanos, herederos del racionalismo en materia de la concepción de sujeto y de derechos, recibe la idea de universalidad, tanto referida al sujeto como a los derechos.

Hemos dicho también que tal construcción del sujeto implica, por una parte, que la subjetividad del sujeto mujeres no ha sido adecuadamente recepcionada, quedando concebida como alteridad, y por otra, se ignora la materialidad de los sujetos invisibilizando el orden social sexual.

En la revisión del derecho internacional pudimos establecer que, con posterioridad a la dictación de la CEDAW, se han producido cambios importantes referidos al problema de discriminación y derechos de las mujeres: se han dado pasos que permiten dar cuenta de modo más sustancial de la subjetividad y materialidad del sujeto mujeres. Sin embargo, estos cambios no han cuestionado de un modo orgánico y sistemático la matriz del sujeto abstracto y universal sobre el cual descansa el sistema de derechos y sus principios fundantes, de igual manera que no han logrado incorporar el análisis en relación con la estructuración social que constituye la base de la discriminación. Lo anterior significa que en el sistema de derechos humanos coexisten el «sustrato original» racionalista y liberal y las nuevas concepciones, en una relación de la cual el sistema no da cuenta, por lo cual tampoco ha creado mecanismos institucionales y jurídicos que permitan superar esta ambivalencia.

El iusfeminismo plantea que hay que avanzar en propuestas que permitan desarticular los mecanismos jurídicos que, derivados de los conceptos construidos a espaldas de la diferencia sexual, conforman estructuras de género y validan estructuras de poder en el derecho. Ello implica, fundamentalmente, avanzar en la recepción de la diferencia sexual y su materialidad en la noción de discriminación y luego, sobre dicha construcción, rearticular (deconstruir y reconstruir) las concepciones estructurantes del sistema de derechos, como los principios que lo fundan, sus normas interpretativas, la configuración de los derechos, el sistema de garantías y, lo más

15. Al respecto, Yanira Zúñiga señala: «Sin perjuicio de la progresiva extensión a otras áreas normativas $[\ldots]$, estos derechos siguen siendo eminentemente precarios, sobre todo respecto de las mujeres. Si bien el seguimiento internacional de las conferencias internacionales [...] ha arrojado avances especialmente notables en la vertebración de una institucionalidad de género [...], también ha dado cuenta que dicha arquitectura no ha supuesto grandes avances en el reconocimiento normativo y en la garantía de los [derechos sexuales y reproductivos], especialmente en Latinoamérica» (Zúñiga, 2012: 4-5). 
fundamental, la subjetivización del sujeto titular de derechos (humanos).

En este planteamiento tiene sentido la noción de discriminación estructural, la que justamente se construye a partir de los elementos materiales que configuran el orden social que implica la subordinación de las mujeres y su discriminación en materia de derechos.

El género es un mecanismo que estructura las relaciones de poder entre lo femenino y lo masculino, que coloca a mujeres y hombres en diferentes posiciones dentro de la estructura social, conformando grupos de pertenencia social (estatus). El planteamiento feminista postula que la discriminación del sujeto mujeres se funda en la diferencia de estatus por razones de sexo. Las desigualdades son el resultado de una inadecuada distribución del poder social, lo que se expresa también en el orden jurídico, puesto que la discriminación jurídica no es diferente a la discriminación social (Bodelón, 1998: 14).

Es importante distinguir entre el fenómeno de discriminación de trato y la discriminación de estatus, es decir, diferenciar entre la discriminación como una ruptura de la igualdad de trato, introduciendo una diferencia antijurídica entre o respecto de individuos que pertenecen a un mismo grupo, y la ruptura de la igualdad respecto o entre sujetos que pertenecen a grupos con diferente poder social (Barrère, 2007: 4-7).

Lo anterior permite plantear el constructo discriminación estructural para referirse a la realidad de discriminación sistemática que viven grupos de sujetos producto de la estructuración del orden social. Barrère ha configurado un concepto aun más específico, al que ha llamado subordiscriminación. Esta noción sintetiza y expresa que la discriminación de género es producto de la subordinación sistémica de las mujeres y permite diferenciar los actos de discriminación de estatus de actos discriminatorios fundados en la diferencia de trato (Barrère, 2001: 39).

Es importante precisar que los casos que el derecho de matriz liberal califica como discriminación son, en realidad, epifenómenos de la discriminación estructural, y se expresan como hechos individuales, pasando por alto que en realidad son una manifestación de la subordiscriminación. Con ello, el derecho provoca una selección de la realidad, que se reduce solo a aquella que se manifiesta como discriminación de trato.

Son el orden social y las relaciones de poder establecidas los que configuran sistémicamente la discriminación, porque lo discriminatorio es el orden social en sí, que se expresa en lo jurídico. Así, se están configurando o reproduciendo sistemáticamente los actos discriminatorios, que son las normas sociales que configuran estereotipos y roles que alimentan los sistemas de opresión (Barrère, 2001: 40). El planteamiento de la discriminación estructural o subordiscriminación no se refiere a una circunstancia que afecte a los sujetos individualmente, sino que a relaciones de dominación que afectan a un grupo específico de sujetos (Barrère, 2003: 6), todos quienes forman parte del grupo sufren discriminación. En otras palabras, las mujeres participan de una situación determinada en función del género que les es común a 
todas, más allá de cualquier otra diferencia (de clase, étnica, racial, de opción sexual, nacionalidad) que las pueda afectar. En este sentido, las mujeres constituyen un grupo socialmente definido (estatus), que - usando el concepto de Barrère- es víctima de subordiscriminación. Young señala expresamente que la opresión es una condición de grupos. Un grupo social se define fundamentalmente por un sentido de identidad, por la identificación con «cierta categoría social», que está dado por una historia común que la genera, y la autoidentificación, que es lo que permite definir a un grupo como grupo social. Siguiendo a Young, este grupo social podrá ser calificado de oprimido (subor-discriminado) si está presente al menos una de las siguientes condiciones: explotación, marginación, carencia de poder, imperialismo cultural y/o violencia (Young, 2000: 77-84). Así planteado, desde la subordiscriminación es posible explicar o dar cuenta de fenómenos estructurales de discriminación que afectan a las mujeres como grupo social.

En el modelo de discriminación estructural los actos de discriminación no son imputables en su origen a sujetos o instituciones, ya que se fundan en un orden social y en las relaciones de poder que en él se constituyen (Bodelón, 2010: 90). No son resultado de decisiones, elecciones o políticas; de hecho, no es necesario la conciencia o intencionalidad de oprimir, sino que basta con que un grupo social se beneficie con la opresión del otro.

Respecto del sistema de derechos, la rearticulación de la noción de discriminación basada en la subordinación estructural de las mujeres indica que debe someterse a un proceso de deconstrucción y reconstrucción de las nociones y contenidos fundamentales, para superar el sesgo de género que aún permanece en el sistema de derechos humanos. Veremos cuál es la propuesta iusfeminista al respecto.

\section{Derechos humanos y discriminación estructural}

Analizar los derechos desde esta perspectiva supone dos desafíos. Por una parte, abordar la reconfiguración de los derechos ya establecidos a partir de la consideración de la realidad material de las mujeres, y, por otro lado, partiendo de esta misma realidad, plantearse la configuración sistémica de derechos humanos que se requieren para abordar subjetividades específicas del sujeto mujeres que gravitan como factor de discriminación. Estos son los derechos sexuales y reproductivos y el derecho a un mundo libre de violencia.

La consideración de las mujeres sobre la base de su materialidad requiere la reconfiguración de derechos ya determinados, integrando las subjetividades sobre la base de la diferencia sexual. Básicamente, se trata de redefinir el contenido y alcance de los derechos fundamentales, tomando en consideración la realidad de las mujeres, en particular en lo que se refiere a la configuración de relaciones de subordinación y su consecuencia de discriminación en la titularidad, goce, ejercicio y garantía de sus 
derechos. Usando la terminología del jurista De Cabo Martín (2006), estos derechos deben ser dotados de historicidad, de materialidad. Así, en el caso de los sujetos que nos ocupan, el trabajo de cuidado y la reproducción, la división sexual del trabajo, la corresponsabilidad, la violencia de género, las barreras a la ciudadanía de las mujeres, la autonomía y libertad de las mujeres, la heterodesignación, la constitución de las mujeres en sujeto de derecho pleno y en igualdad (de condiciones) deberán ser elementos determinantes del contenido de los derechos fundamentales. Todos y cada uno de los derechos deben ser reescriturados a la luz de aquellos elementos de la historicidad/materialidad de los sujetos que permitan «superar» la subordiscriminación que limita los derechos y/o su ejercicio. Es necesario generar un cambio que permita la creación de un sistema de derechos (incluidas las garantías primarias y secundarias) constituido en su integralidad sobre la base del reconocimiento de la diversidad de los sujetos y configurado para asegurar la titularidad, goce y garantía a todos sin discriminación. Usando como ejemplo un tema paradigmático en materia de discriminación de género como es la violencia de género, junto con la consagración del derecho a un mundo libre de violencia, se deberán abordar otros derechos fundamentales, incorporando en su conceptualización elementos que permitan de un modo coherente, sistemático y efectivo garantizar el derecho a un mundo libre de violencia. Especial importancia tiene en esta materia el derecho a la vida y la integridad física y psíquica, el cual debe incorporar la conceptualización de la protección del derecho a la vida desde una perspectiva grupal de género. Además, debe configurarse una normativa referida a la prevención y erradicación de la violencia de género, que permita la garantía de los derechos establecidos, creando una institucionalidad normativa que vehiculice las medidas para la prevención y la erradicación, garantizando el acceso a la justicia: procedimientos procesales, normas sobre judicatura y la creación de sistemas de denuncia judicial y policial. Se debe garantizar también en esta materia el derecho a la salud, que implica prevención y atención, particularmente en materia de salud mental, y, por último, se debe realizar una revisión transversal de los contenidos de los derechos referidos al sistema educativo, a fin de eliminar aquellos de carácter sexista, que se funden en o reproduzcan estereotipos de las mujeres.

En cuanto a la articulación vertebrada de nuevos derechos, los derechos sexuales y reproductivos tienen una importancia central, ya que se anclan en el derecho de las mujeres sobre su propio cuerpo, en el sentido de la afirmación de un espacio de autodeterminación de su persona, lo que implica su libertad sexual y su libertad reproductiva. La estructuración de estos derechos desde la autodeterminación es central respecto del reconocimiento de los derechos y subjetividad de las mujeres, ya que, por una parte, la capacidad procreativa es un rasgo propio y específico de éstas $y$, por otra, a partir de esta característica biológica se ha construido un conjunto de estereotipos y roles femeninos que configuran de un modo importante la realidad de subordiscriminación. 
Estos derechos deben ser reconocidos de modo específico como un derecho del derecho internacional de los derechos humanos, así como consignados constitucionalmente a nivel nacional. Deben ser planteados como un derecho a la autodeterminación en materia sexual y reproductiva, particularmente en esta última, en cuanto al reconocimiento de la libertad y autonomía para decidir respecto de la reproducción, gestación y parto. La teoría feminista plantea que a las mujeres se les debe reconocer la autodeterminación respecto de su propio cuerpo, que no puede ser concebido como un medio para conseguir fines ajenos, cosificando los cuerpos de las mujeres, negando su condición de sujetos autónomos y su capacidad de construir su propio proyecto de vida. Este derecho se funda en una libertad positiva, que consiste en la libertad de las mujeres de engendrar y de gestar, y en una libertad negativa, en el sentido de que no pueden ser obligadas a la reproducción, gestación y parto contra su voluntad. ${ }^{16}$ Dentro de estos derechos, se consideran también los derechos prestacionales referidos a asegurar el acceso a la salud reproductiva, servicios y procedimientos de salud, que permiten (es un derecho que opera como garantía) los derechos de libertad sexual y reproductiva. ${ }^{17}$ Los derechos prestacionales referidos a la salud reproductiva se refieren también a los procedimientos tecnológicos en materia de fecundidad, como reproducción asistida y reproducción subrogada. Asimismo, estos derechos comprenden el acceso a la información y el conocimiento que permita a las personas tomar sus propias decisiones en materia de sexualidad y reproducción.

El iusfeminismo plantea como derecho fundamental el derecho de las mujeres a una vida libre de violencia. La configuración de un derecho específico se plantea considerando la realidad de violencia que sufren las mujeres en las diversas esferas de la sociedad que es manifestación y consecuencia de la desigualdad sistémica en que viven, lo cual atenta contra sus derechos fundamentales al limitar la titularidad, goce, ejercicio y garantías de éstos (Palacios, 2011: 25-27). No es posible avanzar en la verdadera autonomía del sujeto mujeres y en su configuración plena como sujeto de derechos si el derecho ignora la violencia de género, las razones que la determinan y su implicancia para las mujeres. Como se ha señalado, la Convención de Belem do Pará reconoce expresamente el «derecho a una vida libre de violencia, tanto en el ámbito público como en el privado», recibiendo la tesis feminista que plantea que la violencia de género excede la esfera privada, lo que se expresa en figuras como el acoso sexual y el acoso callejero. Agrega, además, que la vida libre de violencia implica «el derecho

16. El derecho al aborto como derecho fundamental se aborda en Pitch (2003), García (2011) y Ferrajoli (2011a).

17. Los derechos prestacionales están recogidos por el sistema de derechos humanos. La CEDAW establece el derecho «a decidir libre y responsablemente el número de sus hijos y el intervalo entre los nacimientos y a tener acceso la información, la educación y los medios que les permitan ejercer estos derechos» (artículo 16 literal e). 
de la mujer a ser libre de toda forma de discriminación», lo que significa asegurar la plena titularidad, goce, ejercicio y garantías de sus derechos y libertades. Finalmente, consigna que una vida libre de violencia significa: «El derecho de la mujer a ser valorada y educada libre de patrones estereotipados de comportamiento y prácticas sociales y culturales basadas en conceptos de inferioridad o subordinación». El sistema general de derechos humanos debe avanzar en la recepción y abordaje de la violencia contra la mujer como fenómeno estructural de modo similar a la Convención de Belem do Pará. Igualmente, debe avanzar en la reconfiguración de otros derechos humanos en el marco de la existencia de la violencia de género como mecanismo de control, subordinación y de discriminación del sujeto mujeres.

\section{Garantías sexuadas}

El sistema de garantías debe reconfigurarse como un modelo de garantías de género a partir de los siguientes elementos: la conflictividad social sexual como determinante del sistema de garantías, la diversidad de los sujetos a partir de la diferencia sexual y la construcción de las garantías en un modelo de derecho objetivo.

El primero debe fundamentarse en el planteamiento de que la sociedad está en conflicto permanente (en este caso de género) y que dicho conflicto debe ser abordado por la institucionalidad e incluso expresado en los instrumentos de garantía. El sistema de garantías debe dar cuenta de las diferencias de poder, de las relaciones de jerarquía y subordinación sistémicas producto de la diversidad sexual. Este sistema debe estructurarse como garantía para resguardar a las mujeres de la discriminación que resulta de las diferencias de poder social sexual y permitir, además, superar los privilegios masculinos propios del orden social. A modo de ilustración, Ferrajoli plantea al respecto: «[Las materialidades] que producen en concreto las múltiples discriminaciones de las mujeres en la vida social, [...] deben ser 'removidas' mediante la introducción de las garantías adecuadas» (Ferrajoli, 2004: 76).

De acuerdo con Ferrajoli, «todo proceso de democratización de los distintos ámbitos de la vida civil ha ocurrido pues, históricamente, a través de la imposición de límites y vínculos legales a las potestades privadas y del establecimiento de garantías primarias y tutelas jurisdiccionales de los derechos fundamentales» (Ferrajoli, 2011 a: 228), los cuales están expuestos a vulneraciones, que pueden ser muy bien observados en la imposición de limites subjetivos a los derechos civiles en dos de estos ámbitos: la familia y la casa, el trabajo y la fábrica.

El segundo elemento, la diversidad sexual, plantea que es necesario considerar la realidad material de los sujetos resultante de la diversidad sexual, es decir, cuál es el lugar social y los roles sociales específicos asignados en función del género. El derecho no puede abordar a los sujetos como entidades indiferenciadas, sino que debe distinguir entre hombres y mujeres que, si bien son iguales en tanto la dignidad hu- 
mana, se han construido y se configuran con subjetividades propias. En el caso de las mujeres se debe hablar más bien de las subjetividades ausentes: las garantías deben posibilitar la expresión de las mujeres como sujeto pleno.

El tercer elemento, el diseño del sistema de garantías, debe necesariamente contemplar «elementos garantistas de naturaleza objetiva», establecidos por y para la sociedad que es quien los exige y a quien se le garantizan. Dichos elementos garantistas deben configurarse respecto de sujetos colectivos, superando así el planteamiento individual de derechos y garantías subjetivas. El sistema de garantías no se estructura desde una concepción de derechos individuales demandables por el sujeto para satisfacer su interés propio, sino que se plantea desde el sujeto colectivo, considerando lo necesario para la trasformación de las condiciones materiales que determinan la discriminación.

\section{Conclusión}

Desde la teoría del género, en el planteamiento iusfeminista se postula que es necesario estructurar un modelo jurídico institucional específico de no discriminación de género, construido sobre la concepción de discriminación estructural, ya que solo así será posible determinar los requerimientos fundamentales para configurar una institucionalidad capaz de desarrollar herramientas conceptuales que den cuenta de la discriminación que afecta a las mujeres y neutralizar las estructuras de poder y jerarquía. Con la discriminación estructural como punto de partida, las soluciones no se orientan hacia el diseño de herramientas para el logro de la igualdad, sino hacia la determinación y compresión de los elementos constituyentes de la subordinación y discriminación de las mujeres.

El modelo planteado requiere no solo reconocer que la discriminación de las mujeres es de carácter estructural, sino que su origen radica en la existencia de un conflicto social de género que la institucionalidad jurídica debe afrontar para avanzar de manera efectiva en materia de igualdad. Esto supone incorporar en el derecho el diseño de una institucionalidad jurídica que, por una parte, dé cuenta y arbitre respecto de los sujetos en el conflicto originado por el sistema sexo-género y, por otra, permita superar los mecanismos de legitimación que otorga el derecho respecto de la subordinación del sujeto mujeres y los mecanismos institucionales y normativos de control sobre éstas y de su contrapartida en los privilegios masculinos.

La reelaboración conceptual desde la teoría jurídica feminista permite avanzar en la superación de la discriminación en derechos del sujeto mujeres, que es condición básica para avanzar hacia la igualdad sustantiva de hombres y mujeres. 


\section{Referencias}

Arnaud-Duc, Nicole (1993). «Las contradicciones del derecho». En Georges Duby y Michelle Perrot (editores), Historia de las mujeres. Tomo 7 (pp. 91-126). Madrid: Taurus.

Barcellona, Pietro (1996). Postmodernidad y comunidad. El regreso de la vinculación social. Madrid: Trotta.

BArrère, Maggy (2001). P«roblemas del derecho antidiscriminatorio: Subordinación versus discriminación y acción positiva versus igualdad de oportunidades». En Revista Vasca de Administración Pública, 60: 145-163.

-. (2003). «Derecho fundamental a la igualdad de trato, discriminación estructural y empoderamiento de las mujeres». Ponencia en Seminario Internacional sobre «La igualdad de género en tiempos de crisis: crisis económica, género y retribución», Palacio Miramar (Donostia-San Sebastián), 2, 3 y 4 de septiembre de 2009, Defensoría para la Igualdad de Mujeres y Hombres de la CAPV/XXVIII, Cursos de Verano UPV/EHU. Disponible en http://bit.ly/2ORQUHN.

-. (2007). Legislación y jurisprudencia antidiscriminatoria por razones de sexo género. Reflexiones de recopilación y de reforma legislativa. Federación Internacional de Mujeres de Carreras Jurídicas. Disponible en http://bit.ly/2Qoy5J1.

Bodelón, Encarna (1998). «La igualdad y el movimiento de mujeres: propuesta y metodología para el estudio de género». Working Paper, 148. Barcelona: Universitat Autònoma.

-. (2009) «La violencia contra las mujeres y el derecho no androcéntrico: perdidas en la tradición jurídica del feminismo». En Patricia Laurenzo, María Luisa Maqueda y Ana Rubio (coordinadoras), Género, violencia y derecho (pp. 221-240). Buenos Aires: Ediciones del Puerto.

-. (2010). «Las leyes de igualdad de género en España y Europa: ¿Hacia una nueva ciudadanía?». Anuario de Filosofía del Derecho, 26: 85-106.

De Cabo Martín, Carlos (2001). «El sujeto y sus derechos». Revista de Teoría y realidad constitucional, 7: 117-135.

-. (2006). Teoría constitucional de la solidaridad. Madrid: Marcial Pons.

Dimier, Alwin (1985). «Los derechos humanos desde una perspectiva europea». En Varios autores, Los fundamentos filosóficos de los derechos humanos (pp. 106-125). Barcelona: Serval y Unesco.

Dippel, Horst (2009). Constitucionalismo moderno. Madrid: Marcial Pons.

Ferrajoli, Luigi (2004). Derechos y garantías. La ley del más débil. $4 \cdot^{\mathrm{a}}$ edición. Madrid: Trotta.

-. (2011a). Principia iuris. Teoría del derecho y la democracia. 2. Teoría de la democracia. Madrid: Trotta.

—. (2011b). «El principio de igualdad y la diferencia de género». En Juan Cruz Par- 
cero y Rodolfo Vásquez (coordinadores), Debates constitucionales sobre derechos humanos de las mujeres (pp. 1-26). Colección Género Derecho y Justicia. México: Suprema Corte de Justicia de la Nación y Editorial Fontamara.

Fraisse, Geneviève (2003). Los dos gobiernos: La familia y la ciudad. Instituto de la Mujer, Universitat de Valencia. Madrid: Cátedra.

García, Cristina (2011). «Autonomía de las mujeres y derechos reproductivos». Jueces para la Democracia, 71.

Habermas, Jürgen (2008). Facticidad y validez. Sobre el derecho y el Estado democrático de derecho en términos de teoría de discurso. $5 .^{\text {a }}$ edición. Madrid: Trotta.

Hespanha, Antonio Manuel (2002). Cultura jurídica europea. Síntesis de un milenio. Madrid: Tecnos.

Mathieu, Vittorio (1985). «Prolegómenos a un estudio de los derechos humanos desde el punto de vista de la comunidad internacional». En Varios autores, Los fundamentos filosóficos de los derechos humanos (pp. 34-51). Barcelona: Serval y Unesco.

Medina, Cecilia (2006). «Observación General 16 del Comité de Derechos Económicos, Sociales y Culturales: Mujer e Igualdad». Anuario de Derechos Humanos, 2011: 127-132. DOI: 10.5354/0718-2279.2011.13379.

Mestre, Ruth (2011). «La ciudadanía de las mujeres: El espacio de las necesidades a la luz del derecho antidiscriminatorio y la participación política». Anales de la Cátedra Francisco Suárez, 45: 147-166.

ONU (1947). «Report of the 1st session of the Commission on the Status of Women to the Economic and Social Council (E/281/Rev.1), Economic and Social Council, 25 February 1947». Disponible en http://bit.ly/2JPoclb.

-. (1995). «Declaración y Plataforma de Acción de Beijing, aprobadas en la Cuarta Conferencia Mundial sobre la Mujer, Beijing, 4 a 15 de septiembre de 1995». Disponible en http://bit.ly/2RHNQLt.

Palacios, Patricia (2011). El tratamiento de la violencia de género en la organización de Naciones Unidas. Santiago: Universidad de Chile. Disponible en http://www. repositorio.uchile.cl/handle/2250/122738.

Pateman, Carole (2000). «El Estado de bienestar patriarcal». Contextos, 2, 5. Programa de Estudios de Género Pontificia Universidad Católica del Perú, Lima.

Peces-BArba, Gregorio (1995). Curso de derechos fundamentales: Teoría general. Madrid: Boletín Oficial del Estado.

Piтch, Tamar (2003). Un derecho para dos. La construcción jurídica de género, sexo y sexualidad. Madrid: Trotta.

Rubio, Ana (2006). «Ciudadanía y sociedad civil: Avanzar en la igualdad desde la política». En Ana Rubio y Joaquín Herrera (editores), Lo público y lo privado en contexto de la globalización (pp. 23-66). Junta de Andalucía, Instituto Andaluz de la Mujer. 
Vitale, Ermanno (2009). «¿Teoría general del derecho o fundación de una república óptima? Cinco dudas sobre la teoría de los derechos fundamentales de Luigi Ferrajoli». En Antonio de Cabo y Gerardo Pisarello (editores), Los fundamentos de los derechos fundamentales. $4^{\text {a }}$ edición. Madrid: Trotta.

Young, Iris Marion (2000). La justicia y la política de la diferencia. Madrid: Cátedra. ZúÑIGA, Yanira (2012). La regulación del aborto: Entre el control y la autonomía. Santiago: Universidad Diego Portales, Facultad de Derecho. Disponible en http://bit. ly/2AuQRsK.

\section{Sobre la autora}

Claudia Iriarte Rivas es licenciada en Ciencias Jurídicas (abogada) de la Facultad de Derecho de la Universidad de Chile, doctora en Derecho y profesora invitada de la Cátedra Género y Derecho en la misma universidad. Su correo es ciriarter@derechouchile.cl. 
El Anuario de Derechos Humanos es una publicación anual de referencia y consulta en derechos humanos y materias afines, que busca ser un espacio de discusión, difusión y conocimiento de los temas centrales sobre derechos humanos en sus contextos nacional e internacional, poniendo a la disposición del público de manera gratuita los distintos desarrollos doctrinales, jurisprudenciales y legislativos ocurridos en este campo dentro del período anual cubierto por cada edición.

\author{
DIRECTORA RESPONSABLE \\ Nancy Yáñez Fuenzalida \\ EDITOR DE CONTENIDOS \\ Salvador Millaleo \\ SITIO WEB \\ anuariocdh.uchile.cl \\ CORREO ELECTRÓNICO \\ anuario-cdh@derecho.uchile.cl
}

LICENCIA DE ESTE ARTÍ́CULO

Creative Commons Atribución Compartir Igual 4.0 Internacional

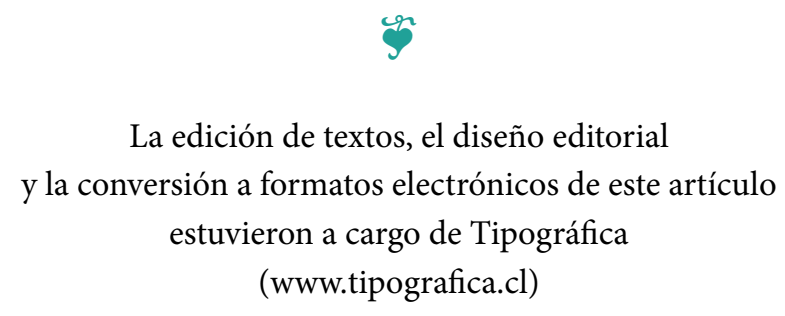

